\title{
A intervenção da fisioterapia no tratamento da incontinência urinaria de esforço
}

\author{
Physical therapy intervention in the treatment of stress urinary incontinence \\ Intervencíon de fisioterapia em el tratamiento de la incontinência urinaria de esfuerzo
}

Recebido: 13/09/2021 | Revisado: 17/09/2021 | Aceito: 20/09/2021 | Publicado: 22/09/2021

\author{
Marta Maiara Silva Olivetto \\ ORCID: https://orcid.org/0000-0003-2180-2304 \\ Centro Universitário da Amazônia, Brasil \\ E-mail: jwmmaaraolivetto@gmail.com \\ Brenda Ellen da Silva Lima \\ ORCID: https://orcid.org/0000-0003-4288-0942 \\ Centro Universitário da Amazônia, Brasil \\ E-mail: limab5807@gmail.com \\ Indiara de Alencar \\ ORCID: https://orcid.org/0000-0003-3204-3278 \\ Universidade do Estado do Pará, Brasil \\ E-mail: indiara.alencarstm@gmail.com
}

\begin{abstract}
Resumo
A incontinência urinária (IU) pode ter várias causas para a sua manifestação no organismo, tais como: características anatômicas diferentes e problemas neuromusculares e pode acarretar impactos psicossociais às pessoas acometidas, sendo em sua maioria o público feminino. Identifica-se como objetivo desse trabalho, compreender a importância da atuação do profissional fisioterapeuta no atendimento de mulheres com incontinência urinária de esforço. Trata-se de uma revisão narrativa de literatura, cuja finalidade é permitir que o resumo possa atualizar e obter informações acerca de um determinado tema em curto período de tempo. A pesquisa foi realizada nas principais bases de dados da área da saúde: Latino-Americana e do Caribe em Ciências da Saúde (LILACS), Scientific Electronic Library Online (SCIELO), PUBMED e Google Scholar. Diante disso, através dessa pesquisa foi possível visualizar a fisioterapia como uma das formas de tratamento e também de prevenção que proporcionam melhor relação risco-benefício para as mulheres acometidas pela IU. O recurso fisioterapêutico como a cinesioterapia é um tratamento positivo, pois viabiliza o desenvolvimento e a melhora da qualidade de vida de pacientes. Outro recurso, é a eletroestimulação que promove dependendo dos seus parâmetros adequados para patologia uma melhora significativa na perda involuntária de urina. Com isso, os três recursos sendo o biofeedback, cones vaginas e eletroestimulação se tornam aliados no tratamento e fortalecimento de toda região e musculatura pélvica. Por fim, compreende-se que o profissional fisioterapeuta e seus recursos tem um papel fundamental no auxílio do tratamento de paciente com incontinência urinária principalmente para melhorar a qualidade de vida e o fortalecimento do assoalho pélvico, e trazer novamente o conforto para poder realizar as tarefas diárias.
\end{abstract}

Palavras-chave: Fisioterapia; Incontinência urinária; Promoção da saúde.

\begin{abstract}
Urinary incontinence (UI) can have several causes for its manifestation in the body, such as different anatomical characteristics, neuromuscular problems and psychological problems, which exacerbate women's health. Stress incontinence is the most affected by women in different age groups. In relation to this, it happens because there is still no way to avoid daily physical or physiological efforts by the body. In this context, stress urinary incontinence is defined as any non-voluntary loss of urine during moments of coughing, physical activities, sneezing, as well as exercises that promote rapid movement of position. The objective of this work is to discuss the importance of the role of the professional physiotherapist in the care of women with stress urinary incontinence. The physical therapy resource such as kinesiotherapy is a positive treatment, as it enables the development and improvement of patients' quality of life. Another resource is the electrical stimulation that promotes, depending on its parameters suitable for the pathology, a significant improvement in the involuntary loss of urine. With this, the three resources being biofeedback, vaginal cones and electrostimulation become allies in the treatment and strengthening of the entire region and pelvic muscles. Finally, it is understood that the physiotherapist and its resources have a fundamental role in helping the treatment of patients with urinary incontinence, mainly to improve the quality of life and strengthen the pelvic floor, and bring back the comfort to be able to perform the tasks daily.
\end{abstract}

Keywords: Physical therapy Specialty; Nocturnal enuresis; Health promotion.

\section{Resumen}

La incontinencia urinaria (IU) puede tener varias causas para su manifestación en el organismo, como diferentes características anatómicas, problemas neuromusculares y problemas psicológicos, que agravan la salud de la mujer. 
La incontinencia de esfuerzo es la más afectada por mujeres de diferentes grupos de edad. En relación a esto, sucede porque todavía no hay forma de evitar los esfuerzos físicos o fisiológicos diarios del cuerpo. En este contexto, la incontinencia urinaria de esfuerzo se define como cualquier pérdida no voluntaria de orina durante los momentos de tos, actividades físicas, estornudos, así como los ejercicios que promueven el movimiento rápido de posición. El objetivo de este trabajo es discutir la importancia del papel del fisioterapeuta profesional en el cuidado de la mujer con incontinencia urinaria de esfuerzo. El recurso de la fisioterapia como la kinesioterapia es un tratamiento positivo, ya que permite el desarrollo y mejora de la calidad de vida de los pacientes. Otro recurso es la estimulación eléctrica que promueve, dependiendo de sus parámetros adecuados a la patología, una mejora significativa en la pérdida involuntaria de orina. Con esto, los tres recursos que son el biofeedback, los conos vaginales y la electroestimulación se convierten en aliados en el tratamiento y fortalecimiento de toda la región y la musculatura pélvica. Finalmente, se entiende que el fisioterapeuta y sus recursos tienen un papel fundamental en ayudar al tratamiento de los pacientes con incontinencia urinaria, principalmente para mejorar la calidad de vida y fortalecer el suelo pélvico, y devolver la comodidad para poder realizar las tareas diarias.

Palabras clave: Fisioterapia; Enuresis nocturna; Promoción de la salud.

\section{Introdução}

A incontinência urinária (IU) pode ter várias causas para a sua manifestação no organismo, tais como: características anatômicas diferentes e problemas neuromusculares o que pode gerar acometimentos psicológicos e sociais modificando de forma exacerbada a saúde da mulher, que é o público mais afetado pela disfunção referida. As pacientes com o passar do tempo por se sentirem constrangidas e com falta de informações necessárias, desconhecem a patologia e acabam convivendo com o problema que vai se agravando cada vez mais e afetando mais ainda sua qualidade de vida (Oliveira \& Garcia, 2011).

A IU é classificada pela Internacional Continence Society (ICS) como qualquer perda de miccional involuntária, é necessário ser baseada no tipo, frequência ou intensidade, fatores predisponentes, influência social, condição de saúde, influência dos hábitos diários e os métodos de aplicação que inibem a micção. O diagnóstico de IU é clínico, com base na história clínica, contém todas as informações e exame físico detalhado (Carvalho \& Freitas, 2011).

A incontinência urinária de esforço (IUE) é a que mais acomete mulheres em diversas faixas etárias. Em relação a isso, acontece, pois, ainda não existe uma maneira de se evitar esforços diários tanto físicos ou fisiológicos do organismo (Marques \& Ferreira 2011). Nesse contexto, a ICS define como incontinência urinaria de esforço como toda perda de urina de forma não voluntaria durante momentos de tosse, atividades físicas, espirros, como também exercícios que promovam movimentação rápida de posição, com isso, a IUE pode ser caracterizada urodinamicamente, quando existe eliminação de urina não planejada pela ascensão de pressão intravestical, ultrapassando a tensão que a bexiga pode suportar (Felicíssimo et al., 2007).

Sabe-se que o enfraquecimento do assoalho pélvico é um dos fatores que pode levar ao surgimento da IU. O assoalho pélvico feminino está separado em partes, sendo na parte frontal a bexiga e a uretra, na parte medial a vagina e na região posterior o reto. Nesse contexto, a pelve feminina é constituída por estruturas de sustentação: diafragma pélvico e urogenital e as fáscias pélvicas. Dessa maneira, na sua organização em grande quantidade é de fibras de contração lenta e em menor quantidade de contração rápida, com isso, todas as partes são de grande relevância tanto no suporte e na manutenção das estruturas pélvicas e suas composições fisiológicas (Spence, 1991).

O tratamento para IU pode ser tratado na modalidade conservadora ou cirúrgico, na alternativa cirúrgica o custo para realização é elevado por ser um procedimento invasivo, existe a possibilidade de ocorrer complicações e ser recusado pelo próprio organismo, além das contraindicações. Nessa análise, o papel da fisioterapia é atuar com mecanismos menos invasivos como a eletroestimulação, biofeedback, e a terapia com cones, aliados a isso, para melhor prevenir e intervir na patologia. O exercício mais eficaz é realizado com o auxílio de um fisioterapeuta, este tipo de técnica envolve a contração controlada e sistemática dos músculos do assoalho pélvico permitindo o aumento na capacidade dos grupos musculares de se contraírem 
automaticamente, com isso, eles são usados para fortalecer os músculos pélvicos e pode-se incluir um plano de atividade física, incluindo exercícios proprioceptivos e procedimentos específicos para os músculos pélvicos (Moreno, 2004).

Identifica-se como objetivo desse trabalho, compreender a importância da atuação do profissional fisioterapeuta no atendimento de mulheres com incontinência urinária de esforço. Dessa forma. Alguns pontos importantes sobre anatomia, fisiologia e etiologia da IU foram adotados e relacionados às técnicas de fisioterapia mais utilizadas nos quadros de IU.

\section{Metodologia}

Trata-se de uma revisão narrativa de literatura, cuja finalidade é permitir que o resumo possa atualizar e obter informações acerca de um determinado tema em curto período de tempo. Assim, consiste em uma publicação que contempla o "estado da arte" da temática proposta, ou seja, constrói-se a partir de análises de material bibliográfico já publicado sobre o tema escolhido (Rother, 2007).

Para a construção deste trabalho buscou-se estudos publicados que apresentam relevância no meio científico sobre a temática proposta, seguindo normas fases de preparação, desenvolvidas da seguinte forma: elaboração da questão problema, busca dos artigos, coleta de dados, análise crítica dos estudos inclusos e discussão dos resultados apresentados, com isso, abordar de maneira objetiva e coesa um acervo significativo de artigos científicos, os quais se dão por essência deste trabalho.

A pesquisa foi realizada nas principais bases de dados da área da saúde: Latino-Americana e do Caribe em Ciências da Saúde (LILACS), Scientific Electronic Library Online (SCIELO), PUBMED e Google Scholar, utilizando os descritores Fisioterapia, incontinência urinaria e promoção de saúde localizados na lista dos Descritores em Ciências da Saúde. Foram selecionados como critérios de inclusão: artigos completos disponíveis integralmente nas bases de dados elencados, em idiomas português, espanhol e inglês e relacionados com a relevância do tema. Foram excluídos artigos duplicados, incompletos, resumos, resenhas, debates, artigos publicados em anais de eventos e indisponíveis na íntegra.

Então, nessa perspectiva, para direcionar a construção dessa revisão delineou-se como a questão problema principal: “Qual é o papel da fisioterapia no tratamento de pacientes com incontinência urinaria de esforço?". E estipula-se como objetivo geral: Compreender a importância da intervenção fisioterapêutica nas suas mais variadas formas no tratamento e prevenção da IU.

\section{Resultados e Discussão}

A partir das pesquisas realizadas foi possível descrever de forma exploratória acerca da temática escolhida e estabelecer alguns pontos principais para serem expostos e discutidos, sendo eles: o primeiro trata sobre as estruturas anatômicas e funções orgânicas envolvidas na incontinência urinária. $\mathrm{O}$ segundo descreve a incontinência urinária nas suas mais variadas e possíveis formas de manifestação. $\mathrm{O}$ terceiro ponto a ser exibido são as técnicas fisioterapêuticas utilizadas no tratamento das mais diversas formas de incontinência urinária.

\section{Anatomia pélvica feminina: estruturas e funções envolvidas na incontinência urinária}

A anatomia pélvica é caracterizada por tecido conjuntivo, ligamentos, ossos e músculos, seu principal trabalho é para contenção das vísceras pélvicas e as abdominais, viabilizando o trajeto da uretra, reto e vagina, com isso, funcionar de forma correta na parte reprodutora e postural (Ramos, 2014).

A organização pélvica feminina é definida como uma estrutura grande com forma arredondado e uma disposição óssea que se estreita. Sua concretização está relacionada com dois ossos do quadril são eles, o sacro e o cóccix que são formados pelos ossos púbis, ílio e ísquio. Nesse contexto, essas estruturas articulam-se na parte anterior com a sínfise púbica e 
posteriormente no sacro, por intermédio das articulações sacrilíacas. Dessa maneira, os ligamentos presentes na pelve são distribuídos entre o sacro e as outras composições pélvicas como, ilíaco, ísquio, cóccix e os ossos púbicos (Portugal \& Palma, 2009).

Toda a organização que está disposta no perímetro do peritônio e a pele da vulva como, a uretra, os músculos da pelve e a bexiga dão origem ao assoalho pélvico, na qual é formada por fáscias, músculos, ligamentos que proporcionam a sustentação dos músculos abdominais. A depressão entre o abdome e a pelve está conectada, porém a região inferior é distanciada por um conjunto de músculos-fáscias estabelecendo a formação de um diafragma pélvico, que é definido como uma estrutura formada por músculos do coccígeo e ânus que integram inferiormente a cavidade pélvica, na qual tem a responsabilidade de apoiar as vísceras pélvicas, especificamente o útero (Rubinstein et al., 2000).

\section{Bexiga Urinária}

A bexiga é uma estrutura muscular parecida com um saco oco e elástico situado na parte posterior a púbis. Nessa análise, ela é constituída por duas vias de entrada, definidas como ureteres e uma via de saída, denominada como junção uretrovesical. É distanciada por níveis de camadas de músculos como a camada lisa, definhada como o musculo detrusor. Na região superior esses níveis de camadas se juntam e suas composições de fibras podem alterar a direção e o plano, sendo um fator necessário para um bom esvaziamento vesical. Nesse contexto, o esvaziamento acontece devido a contração muscular do detrusor e o relaxamento dos esfíncteres uretrais. A bexiga pode suportar dentro do seu saco a quantidade entre 300 a $600 \mathrm{ml} \mathrm{de}$ urina, e atinge a sua totalidade de urina antes da sua capacidade total, gerando a micção. A atividade exercida pela bexiga recebe ordens de um sistema complexo de nervos que se estabelecem no trígono e estruturas como, o trato urinário, sistema nervoso central e periférico. É a ligação entres essas composições que permite um equilíbrio harmônico definido com continência urinária (Smeltzer \& Bare, 2005).

\section{Uretra}

A uretra é um tipo de ducto muscular que transporta a urina do ósito interno da uretra da bexiga até o ósito externo. Sua estrutura é em camadas, na qual o nível muscular é combinado entre musculo estreado e o liso. A submucosa tem como foco a continência urinaria e a mucosa, uma estrutura de camada epitelial que inibi a luz uretral, menos a micção. Nesse contexto, na junção da uretra com a bexiga, parte da bexiga cerca a uretra e estabelece a função de esfíncter interno para manter o sistema fechado, no tempo de miç̧ão contrai-se e as alterações resultantes por meio da contração realizada é a abertura do esfíncter e o repouso a decida do colo vesical é normal no início da micção (Oliveira \& Garcia, 2007).

\section{Assoalho Pélvico}

A estruturação do assoalho pélvico é composta por estruturas de músculos e fáscias que estão na parte perineal. Nesse cenário, a região do períneo é uma divisão elencada com formato de losango que se dispõe na parte inferior da cavidade da pelve, com isso, as superfícies que demarcam o períneo são a sínfise púbica, ramos da parte de baixo do púbis, ramificação isquiopúbicos da região do quadril, ligamentos sacrotuberais, túberes isquiáticos, região inferior do cóccix e do sacro (Moreno 2009).

A formação do assoalho pélvico tem como função proporcionar a contenção, suporte e suspensão aos órgãos localizados na pelve como os ovários, útero, bexiga e a região terminal do intestino, com participação de grande relevância no processo de continência, excreção e sexual. Nessa circunstância, sua estrutura de apoio é por meio de fibras de contração lenta do tipo 1, que preserva o tônus muscular, facilitando o colo na posição elevada e que pode se alterar pela contração vigorosa ou 
de repouso. Em contrapartida, as fibras de contração rápida do tipo 2, obtém rápidas respostas de contratilidade (Spence, 1991).

Alguns autores definem o assoalho pélvico como triângulos divididos em duas partes caraterizadas como diafragma pélvico na parte caudal e o urogenital na parte superior. O diafragma da pelve é integrado por fáscias e pelos músculos pubococcígeo, puborretal, iliococcígeo, levantador do ânus e coccígeo, possuindo a atividade anorretal. Com relação, ao diafragma urogenital, ele é constituído por músculos como bubocavernoso, isquiocavernoso, transverso superficial e profundo do períneo, e os esfíncteres uretral e anal. Nessa conjuntura, é definida como a camada externa do diafragma pélvico, possibilitando a funcionalidade sexual e urinária (Galhardo \& Katayama, 2007).

$\mathrm{Na}$ parte lateral do assoalho pélvico existe duas estruturas definidas como arcos tendíneos do elevador do ânus e da fáscia, as duas são formadas por tecido conjuntivo, necessários na sustentação da musculatura do elevador do ânus, uretra e parede vaginal anterolateral. Nessa circunstância, foi evidenciado por autores que o tecido conjuntivo é a base de sustentação em três níveis de camada, a primeira formada por ligamentos útero-sacrais, e cardinais, a segunda por ligamentos da vagina parte medial e a terceira, pela junção da vagina com estruturas que envolvem o elevador do ânus, a uretra, o musculo perineal e o corpo do períneo (Monteiro \& Filho, 2007).

\section{Continência Urinária}

A continência urinária é viabilizada por recursos fisiológicos e estruturais que tem o controle de fechamento da uretra e conseguem suportar a bexiga e a junção uretrovesical. Para que aconteça a continência urinária é necessário que a bexiga esteja estável, assim como também é fundamental uma pressão intra-uretral maior que a pressão na parte interna da bexiga. Nesse caso, para que ocorra o fechamento da uretra é necessário ser efetuado por três mecanismos, o esfíncter interno e externo e a coaptação produzida pelo plexo vascular da submucosa uretral. A base de sustentação é estabelecida pela fáscia púberocervical que está relacionada a musculatura do levantador do ânus (Matheus et al., 2006).

\section{Fisiologia da Micção}

A bexiga comporta urina até que seu volume atinja o nível médio estabelecido, quando se eleva esse acumulo e a pressão da bexiga excede a pressão uretral, ocorre a repleção vesical, está repleção é mediante a receptores localizados na parede da bexiga. No decorre dessas reações o músculo detrusor deve estar inativo, com as fibras musculares da uretra e de todo o assoalho pélvico envolvendo na realização da continência (Impey, 2007).

É evidenciado que estímulos proporcionados pelo córtex cerebral induzem à vontade miccional, a micção acontece por meio do repouso voluntario de toda a estrutura pélvica e da uretra com relação a contração detrusora viabilizando o esvaziamento de urina. Diante disso, a função proporcionada devido os estímulos do sistema nervoso central e por pontos específicos da micção no tronco cerebral e medula espinhal, sendo mais especifico no sitema nervosos parassimpático na região sacral e no sistema nervoso simpático na região toracolombar (Ramos, 2014).

\section{A incontinência urinária e suas variadas formas de manifestação Incontinência Urinária (IU)}

Segundo a Sociedade Internacional de Continência, definem a incontinência urinária como toda perda não voluntária de urina, na qual é uma disfunção do trato urinário inferior e ocorre devido a modificações na fisiologia da micção ou nas composições que são base de suporte dos órgãos que contribuem para uma boa ação miccional. Nessa análise, os fatores de maior presença da fisiopatologia e dos sintomas mais pertinentes são indivíduos do sexo feminino com 50 anos e com o passar 
da idade, excesso de peso, problemas estrogênicos, movimentos estáticos e dinâmicos de estresse muscular, acidente vascular cerebral e traumas (Marques \& Ferreira, 2011).

Uma pesquisa realizada segundo o autor Almeida (2015) pressupõe que 200 milhões de indivíduos possuem a patologia e convivem com ela e entre 15\% e 30\% dessas pessoas estão na faixa etária de 60 anos com algum tipo IU. Existem algumas variações de incontinência urinária podendo ser permanente ou passageira e sendo classificada em: incontinência urinária de esforço (IUE), incontinência urinária de urgência (IUU), incontinência urinária mista (IUM) e incontinência urinária por transbordamento (IUT).

\section{Incontinência Urinária de Esforço (IUE)}

A incontinência urinária de esforço é definida como sintoma principal, a perda de urina involuntária por esforços, como o ato de correr, mudança de posição sentado para o em pé, levantamento de peso, pular, tossir e espirrar. Nesse contexto, frequentemente não ocorre o esvaziamento de urina durante o sono ou repouso. Em virtude disso, a rotina diária da paciente é alterada tanto por limitações físicas e psicológicas, com isso, a mulher começa a tomar precauções na tentativa de evitar os sintomas da patologia, como a redução de ingestão de líquidos, uso de fármacos, o momento de dormir é afetado, a vida sexual, as mulheres limitam-se a não sair mais para eventos, festas, transporte coletivos e viagens com grande percurso, além disso existe um grande gasto com absorvente, aliados a todos esses fatores o convívio social se torna conturbado e menos agradável. Nesse contexto, A IUE pode ser classificada em três tipos: A primeira, com perda de urina discreta que se manifesta quando realizado algum esforço, a segunda quando a perda involuntária da urina é moderada já com problemas de bexiga e uretra caídos e a terceira, é a perda muito elevada de urina, a bexiga e a uretra podem estar no seu padrão normal, porém sem força para contrair deixando-a sempre aberta (Almeida \& Santos, 2009).

A IUE tem como problema principal a diminuição da pressão uretral, que se origina da hipermobilidade, promovendo uma perda de força na uretra e problemas na descida do colo vesical, na qual é dificultada a vascularização na mucosa da uretra pois não irá acontecer o fechamento correto da uretra. Nesse analise, essa danificação ocorre no esfíncter ou alterações na ordem correta das estruturas da uretra. O quadro clinico para um diagnóstico de maneira eficaz é uma relação entre, o relato da paciente, tabelas miccionais, o quanto é afetado a qualidade de vida mediantes a questionários, teste de esforço, exame físico. É levado em consideração, exame de analise sedimentação urocultural e urinária que são realizados para descarta a infecção urinária e aquecer a paciente para avaliação urodinâmica (Nunes \& Resplande, 2009).

O exame urodinâmico é utilizado com a intenção de realizar uma intervenção cirúrgica, quando existe doença neurológica e casos de incontinência urinária mista. Um fator primordial para o tratamento fisioterapêutico é que se houver uma grande deficiência esfincteriana intrínseca, os procedimentos terão resultados baixos, visto que tem problemas em varais partes da uretra, com inervação muscular estreado, lisa e submucosa (Carvalho \& Freitas, 2011).

\section{Incontinência Urinária de Urgência (IUU)}

Nesse tipo de incontinência acontece que a bexiga apresenta irregularidades na sua função e ocorre uma contração não voluntária na musculatura do detrusor na fase do enchimento de urina. Com isso, é desenvolvida uma sensibilidade vesical e hiperatividade do músculo detrusor que leva a imenso desejo de ir ao banheiro. Uma grande característica dessa patologia é pacientes relatarem a ida ao banheiro mais de oito vezes por dia (Rizzi, 2012).

\section{Incontinência Urinária Mista (IUM)}

A incontinência urinária mista é uma junção da incontinência de urgência e a de esforço, com isso, uma hiperatividade do musculo detrusor e uma insuficiência uretral (Gomes, 2010). 


\section{Incontinência Urinária de Transbordamento (IUT)}

Essa incontinência é caracterizada por ausência de desejo miccional, seu problema principal está ligado a obstrução infravesical com aumento do trato urinário e afastamento da função renal. Nessa análise, o quadro clínico para um bom diagnóstico é o relato do paciente, com realização de exames e ultrassom de vias urinarias e rins (Carvalho \& Freitas, 2011).

\section{Técnicas fisioterapêuticas que atuam no tratamento da incontinência urinária \\ Cinesioterapia}

O foco desse recurso fisioterapêutico é o ganho de resistência muscular da uretra e o desenvolvimento com a intenção de melhorar a sustentação do assoalho pélvico, o objetivo é hipertrofia nas fibras musculares estreado do tipo 2 dos diafragmas pélvico e urogenital. Segundo o autor dessa referência, as atividades elaboradas para contração rápida obtiveram $70 \%$ de cura ou evolução da não perda miccional. No entanto, existe alguns pontos fracos desse tratamento como devido o desconhecimento da paciente em contrair de maneira correta os músculos da pelve, algumas vezes começa a contrair o reto abdominal, adutores e glúteo, por isso, é necessário que o profissional fisioterapeuta fique atento para contrações de forma errada (Peattie et al., 1988).

Na prática, existem vários métodos, antes de iniciar a aplicação de uma série de atividades, é importante saber se a contração da musculatura perineal é realizada corretamente. Por isso, é necessário simular a interrupção do fluxo urinário no banheiro feminino ou em decúbito dorsal, inserir o dedo na vagina e contrair, como se para evitar que a urina escorra, se sentir a força de compressão, significa que a contração necessária está em andamento. Nessa análise, o método inicial é realizado por meio de teste de força da manutenção do tônus muscular e contração. Os indivíduos com a patologia são selecionados de acordo com a sua necessidade desde recursos mais simples, que analisa a habilidade de ativar as fibras do musculo do ânus, é levado em analise a capacidade de controlar o jato urinário. Nessa circunstância, pessoas com um grau elevado de IU podem não responder de forma agradável a essa modalidade, ao passo que casos leves e moderados recebem resultados em pouco tempo. Os exercícios sugeridos no tratamento são realizados em pé contra a gravidade com os membros inferiores um pouco flexionados e afastados, mãos na região das nádegas, pressioná-las para baixo e para frente, enquanto se realiza contração da musculatura pélvica. Pode ser feita de outra forma em pé, os membros inferiores afastados e flexionados, mãos sobre o joelho ou as coxas e permanecer em contração estática e sem movimento da musculatura pélvica. Nesse contexto, existe uma outra forma de ser realizado essa atividade pode ser com cotovelos e joelhos apoiados no solo, os braços devem entrelaçar-se um sobre com o outro onde ocorra maior estabilidade e a cabeça possa se apoiar nos braços permitindo o repouso do pescoço sem causar tensão na região cervical e deve ser feita contração isométrica da musculatura pélvica (Caetano \& Tavares, 2004).

Pode-se feita essa atividade em decúbito dorsal, com os quadris levantados, uma perna dobrada, o pé no chão, a outra perna será esticada e elevada, a pessoa levanta os quadris, uma das pernas foi expandida e contraída o períneo, é necessário fazer exercícios com os dois membros. Modifique o plano de exercícios, que é ficar em uma posição plana, prender uma bola na parte interna da coxa, manter o antepé parado, contrair o períneo, relaxar ao retornar e colocar as solas dos pés no chão, este exercício pode ser feito em uma superfície instável (Castro et al, 2008).

Segundo Moreno (2009), a terapia por exercícios visa realizar contração reflexa para o caso de perda de urina, para isso é necessário o conhecimento da área e da contração perineal, devendo ter força muscular de no mínimo nível 2 para atingir os objetivos funcionais. Os órgãos genitais são fracos, reconhecíveis à palpação, com isso, à medida que os exercícios de resolução de problemas progridem, simule a micção, ao tossir, contração reflexa, subir e descer escadas, agachar e carregar peso. 


\section{Eletroestimulação}

As atividades pélvicas ligadas a uma estimulação elétrica promovem que a paciente se torne consciente sobre a existência dos seus músculos do assoalho pélvico e, com isso, ter um maior domínio da função vesical. A estimulação depende do tipo de frequência de corrente utilizada e pode até inibir o músculo detrusor e mediante a isso baixar os números de micções diárias. Aliados a isso, pode ocasionar melhora do ganho de força para o elevador do ânus, viabilizando a passagem de pressão abdominal. Dessa maneira, esse procedimento é demostrado com eficaz no tratamento, pois é pouco invasivo e seus efeitos colaterais são quase nulos, e o estimulo elétrico desenvolve a melhora da pressão intrauretral e ascensão do fluxo sanguíneo para a região pélvica (Polden \& Mantle, 2002).

\section{Biofeedback}

Tem o objetivo de monitorar pelos aparelhos, o funcionamento fisiológico do indivíduo que não esteja na sua condição normal para diferenciar por si próprio. Nesse caso, com pessoas com incontinência urinária, o procedimento realizado é reconhecimento da musculatura esquelética, presente no repouso e contração da uretra e da micção, ou seja, estabelecer uma pré-contração dos músculos da pelve com ascensão da pressão intra-abdominal e elevar a base de sustentação pélvica por aumento do tônus muscular e hipertrofia. Dessa maneira, para melhorar o quadro clinico do paciente, o tratamento utilizando esses recursos possibilita que o indivíduo estimule as respostas eletrofisiológicas da região pélvica de acordo com a sua percepção. Portanto, para um ótimo desempenho o procedimento deve ter exercícios associados, com a intenção de fortalecer e ocorrer a contração correta dos músculos da continência (Arruda et al., 2007).

Com o avanço do controle dos vários grupos de músculos do assoalho pélvico pela utilização do aparelho, a pessoa começa a reestabelecer o comando voluntário das estruturas do seu corpo, fortalecendo os músculos antagonistas e agonistas. Segundo o estudo feito pelo referido autor, foi observado que o biofeedback associado a atividades proporcionados pela fisioterapia houve uma queda de $82 \%$ dos casos de IU (Burgio et al., 1991).

\section{Cones Vaginais}

O tratamento com cones vaginais é um procedimento para completar o desenvolvimento dos resultados obtidos nos exercícios de ascensão da musculatura do assoalho pélvico, pois é recurso com um grau de dificuldade baixo e o valor não é elevado. Neste sentindo, a intervenção cirúrgica está se tornando inadequado para alguns casos, mas a cinesioterapia com auxílio de um fisioterapeuta é cada vez mais utilizada, pois tem resultados positivos na IU. Entretanto, é primordial uma boa avaliação e a escolha certa dos recursos e técnicas para cada tipo de incontinência urinária. Dessa forma, a atuação da fisioterapia no tratamento tem como objetivo desenvolver uma força muscular para promover uma reeducação abdominal e pélvica, com isso, promover uma estabilização para manter uma continência urinária (Oliveira \& Garcia, 2011).

O recurso fisioterapêutico como a cinesioterapia é um tratamento positivo, pois viabiliza o desenvolvimento e a melhora da qualidade de vida de pacientes. Outro recurso é a eletroestimulação que promove dependendo dos seus parâmetros adequados para patologia uma melhora significativa na perda involuntária de urina. Com isso, os três recursos sendo o biofeedback, cones vaginas e eletroestimulação se tronam aliados no tratamento e fortalecimento de toda região e musculatura pélvica (Castro et al., 2010).

Com base na pesquisa realizada, foi possível estabelecer relações em comum entre alguns artigos encontrados e traçar um quadro (Quadro 1) para expor os artigos e seus principais achados para endossar o objetivo deste trabalho, o qual seja: compreender a importância da intervenção fisioterapêutica no tratamento e prevenção das manifestações de IU. 
Quadro 1 - Exposição de artigos que se relacionam de diferentes formas em relação à incontinência urinária, especialmente, em relação às formas de tratamento e prevenção, dando ênfase às intervenções fisioterapêuticas.

\begin{tabular}{|c|c|c|}
\hline Autoria dos artigos & Tipo de pesquisa/Objetivo & Conclusões \\
\hline Brasil et al, 2018. & $\begin{array}{l}\text { Bibliográfico/ Analisar a produção científica } \\
\text { nacional e internacional quanto à relação escores } \\
\text { dos instrumentos ICIQ-UI-SF (Internacional } \\
\text { Consultation on Incontinence Questionnaire - } \\
\text { Urinary Incontinence/Short Form (ICIQ-UI/SF) e } \\
\text { FSFI (Female Sexual Function Index) na } \\
\text { população feminina. }\end{array}$ & $\begin{array}{l}\text { O baixo quantitativo de produções e resultados } \\
\text { encontrados demonstraram que é necessário um } \\
\text { maior aprofundamento sobre a temática, para um } \\
\text { embasamento e qualificação da assistência para } \\
\text { portadoras de incontinência urinária. }\end{array}$ \\
\hline Lopes et al, 2016. & $\begin{array}{l}\text { Campo/ Relatar a criação, experiência de } \\
\text { implantação e atendimento realizado no } \\
\text { Programa de Reabilitação do Assoalho Pélvico } \\
\text { (PRAP), um projeto da Faculdade de } \\
\text { Enfermagem da Universidade Estadual de } \\
\text { Campinas (UNICAMP), desenvolvido em um } \\
\text { centro de saúde de Campinas, São Paulo, Brasil. }\end{array}$ & $\begin{array}{l}\text { As atividades preventivas e de reabilitação do } \\
\text { assoalho pélvico constituem-se áreas de importante } \\
\text { atuação do enfermeiro e iniciativas como a relatada } \\
\text { contribuem para a formação profissional e prática } \\
\text { baseada em evidências. }\end{array}$ \\
\hline $\begin{array}{l}\text { Delarmelindo et al, } \\
2013 \text {. }\end{array}$ & $\begin{array}{l}\text { Campo/ Discutir a experiência de mulheres que, } \\
\text { após falha no tratamento conservador, } \\
\text { consideravam-se sem perspectivas de acesso ao } \\
\text { tratamento cirúrgico. }\end{array}$ & $\begin{array}{l}\text { A experiência do grupo de mulheres com IU sem a } \\
\text { perspectiva de acesso ao tratamento cirúrgico, após } \\
\text { tentativas frustradas com o conservador, não esbarrou } \\
\text { somente na falta de condição clínica, mas também no } \\
\text { descrédito de profissionais médicos sobre a } \\
\text { efetividade do tratamento cirúrgico da IU na vida } \\
\text { daquelas que se reconheciam em condições. Seriam } \\
\text { necessárias políticas públicas voltadas à saúde da } \\
\text { mulher, com intervenções mais incisivas acerca da } \\
\text { prevenção e da reabilitação. }\end{array}$ \\
\hline Filho et al, 2013. & $\begin{array}{l}\text { Bibliográfico/ Analisar os recursos e sua eficácia } \\
\text { na reabilitação do assoalho pélvico no tratamento } \\
\text { dos prolapsos genitais e incontinência urinária. }\end{array}$ & $\begin{array}{l}\text { Os estudos analisados mostraram que o treinamento } \\
\text { do assoalho pélvico pode ser usado no tratamento dos } \\
\text { prolapsos, porém são necessários mais estudos } \\
\text { randomizados para sustentar essa evidência. Para a } \\
\text { incontinência urinária a reabilitação do assoalho } \\
\text { pélvico é eficiente e deve ser o tratamento de } \\
\text { primeira escolha. }\end{array}$ \\
\hline Fitz et al, 2012. & $\begin{array}{l}\text { Campo/ Verificar o efeito da adição do } \\
\text { biofeedback (BF) ao treinamento dos músculos } \\
\text { do assoalho pélvico (TMAP) para o tratamento da } \\
\text { incontinência urinária de esforço (IUE). }\end{array}$ & $\begin{array}{l}\text { A adição do BF ao TMAP para o tratamento da IUE, } \\
\text { aplicado de acordo com o protocolo descrito, } \\
\text { contribui para melhora dafunção dos MAP, redução } \\
\text { dos sintomas urinários e melhora da qualidade de } \\
\text { vida. }\end{array}$ \\
\hline Pinheiro et al, 2012. & $\begin{array}{l}\text { Campo/ Comparar os efeitos das cinesioterapias } \\
\text { com toque digital e com biofeedback para } \\
\text { consciência perineal de mulheres com } \\
\text { incontinência urinária de esforço. }\end{array}$ & $\begin{array}{l}\text { Tanto a cinesioterapia com uso do biofeedback, } \\
\text { quanto a cinesioterapia com toque digital são } \\
\text { excelentes opções de tratamento para ganho de } \\
\text { consciência perineal. }\end{array}$ \\
\hline
\end{tabular}




\begin{tabular}{|c|c|c|}
\hline $\begin{array}{l}\text { Beuntemuller et al, } \\
2011 \text {. }\end{array}$ & $\begin{array}{l}\text { Campo/ Avaliar o efeito dos exercícios perineais } \\
\text { (EP) associados ou não à eletroterapia em } \\
\text { mulheres com incontinência urinária de esforço } \\
\text { (IUE) quanto à contração dos músculos do } \\
\text { assoalho pélvico (MAP). }\end{array}$ & $\begin{array}{l}\text { Os exercícios foram eficazes na melhora da contração } \\
\text { dos MAP em mulheres com IUE, sem diferença entre } \\
\text { o grupo de eletroterapia mais exercícios em relação } \\
\text { ao grupo de exercícios. }\end{array}$ \\
\hline Fitz et al, 2011. & $\begin{array}{l}\text { Campo/ Avaliar o impacto do treinamento dos } \\
\text { músculos do assoalho pélvico (TMAP) na } \\
\text { qualidade de vida (QV) em mulheres com } \\
\text { incontinência urinária de esforço (IUE). }\end{array}$ & $\begin{array}{l}\text { O treinamento muscular do assoalho pélvico } \\
\text { proporcionou melhora significativa na } \mathrm{QV} \text { de } \\
\text { mulheres com IUE. }\end{array}$ \\
\hline Franco et al, 2011. & $\begin{array}{l}\text { Campo/ Comparar os efeitos do tratamento com } \\
\text { eletroestimulação transvaginal (ET) e do nervo } \\
\text { tibial (ENT) sobre a qualidade de vida (QV) e } \\
\text { queixas de perda urinária em mulheres com } \\
\text { bexiga hiperativa. }\end{array}$ & $\begin{array}{l}\text { Houve melhora da QV em ambos os grupos, assim } \\
\text { como uma diminuição das queixas de perda urinária, } \\
\text { entretanto, o grupo que recebeu ET obteve melhora } \\
\text { nos escores em dois domínios do questionário de QV } \\
\text { genérico após o tratamento, que teve limitação por } \\
\text { aspectos físicos e limitação por aspectos emocionais. } \\
\text { O que não ocorreu com o grupo de ENT. }\end{array}$ \\
\hline Menezes et al, 2011. & $\begin{array}{l}\text { Campo/ Analisar a prevalência e interferência da } \\
\text { incontinência urinária sobre a vida diária de } \\
\text { mulheres de um Centro de Saúde em Fortaleza. }\end{array}$ & $\begin{array}{l}\text { A incontinência urinária apresentou alta prevalência, } \\
\text { interferindo negativamente na vida das mulheres. }\end{array}$ \\
\hline
\end{tabular}

Fonte: Olivetto, Lima e Alencar (2021).

Pode-se perceber que a maior parte dos artigos selecionados que estão expostos na tabela tratam-se de trabalhos de campo, sendo ensaios clínicos e estudos randomizados. Também foram escolhidas duas produções bibliográficas. Isso demonstra, e confirmou-se ao longo da pesquisa, que a maioria dos trabalhos produzidos sobre a temática são de campo, evidenciando a preocupação da Fisioterapia como ciência em aperfeiçoar e corroborar a efetividade de suas técnicas e intervenções no que se refere à temática escolhida.

De forma geral, os artigos tratam da IU como um problema de saúde pública que afeta muitas mulheres, e estas por medo, vergonha ou desconhecimento convivem com o problema e acabam permitindo que ele se agrave ainda mais por não buscar uma solução efetiva, o que acaba afetando sobremaneira vários aspectos de suas vidas. Os artigos também têm em comum uma crítica à profissionais de saúde insensíveis à esta disfunção, uma vez que em suas anamneses não dão à devida atenção a possíveis sinais que denotem o problema. Ainda refletem criticamente sobre a escassez do incentivo aos tratamentos conservadores, entre os quais se inclui as intervenções fisioterapêuticas, sendo estes os mais indicados, pois apresentam os melhores resultados e menores riscos e despesas, funcionando não somente como tratamento, mas principalmente, como prevenção.

\section{Conclusão}

A fisioterapia vem ganhando seu espaço dentre as áreas médicas e assim mostrando sua grande importância, principalmente nas recuperações e tratamento de pacientes, foi possível verificar que o profissional fisioterapeuta e seus recursos tem um papel fundamental no auxílio do tratamento de paciente com incontinência urinária principalmente para melhorar a qualidade de vida e o fortalecimento do assoalho pélvico, e trazer novamente o conforto para poder realizar as tarefas diárias, com isso, foi notório que os recursos fisioterapêuticos aliados a exercícios da cinesioterapia e aparelhos são de 
grande relevância para o tratamento da patologia. Entretanto, é difícil resolver este problema na maioria das vezes porque algumas mulheres querem esconder o problema, ou lidam como uma condição do envelhecimento, por falta de informação.

Por fim, considerando a prevenção de problemas musculares do assoalho pélvico, é cada vez mais importante abandonar o hábito de prestar atenção à disfunção quando os sintomas estão somente em fase avançada. Entende-se que com o aumento da expectativa de vida da população, o número de IUE vem aumentando, além disso, a musculatura pélvica costuma ser lesada durante o parto, reduzindo o nível de hormônios da menopausa. Ao contrário de outros músculos do nosso corpo, esses músculos não movem membros ou articulações, por isso não percebem seus movimentos e não tomam medidas para proteger suas funções.

O objetivo da prevenção é realizar exercícios para aumentar a força do esfíncter externo da bexiga, fortalecer os músculos pélvicos, mais especificamente o músculo elevador do ânus, evitar contraturas e manter a tensão muscular. Observase que com o aumento das doenças do trato urinário inferior, alguns aspectos precisam ser mais bem discutidos no âmbito do Sistema Único de Saúde (SUS) para confirmar a importância do fisioterapeuta nos planos de saúde da família para que possa intervir na alta prevalência de incontinência urinaria. É necessário promover o acesso dos indivíduos a esses cuidados e conscientizá-los sobre o papel do profissional nas doenças do trato urinário e promover a o melhor acesso a esses cuidados.

Em virtude disso, deve-se ressaltar que, em relação aos privilégios de que gozam os autores das referências selecionadas nesta revisão de literatura, a escolha de teorias e métodos para realizar inovações pode ser considerada uma abordagem promissora para o desenvolvimento do conhecimento atual. Neste tópico, portanto, recomenda-se o uso de métodos qualitativos em pesquisas futuras e a adoção de teorias mais profundas que possam refletir sobre a experiência emocional e funcional dessa população antes, durante e após as complicações que levam ao desenvolvimento da incontinência urinária.

\section{Referências}

Almeida, A. C., Santos, A. F. C. (2009). Prevalência e Implicações Clínicas da Incontinência Urinária em Mulheres de uma Unidade Básica de Saúde em Belém do Pará. Trabalho de Conclusão de Curso (Graduação) - Centro de Ciências Biológicas, curso de Fisioterapia, Universidade da Amazônia, Belém do Pará.

Almeida, F. \& Marsal, A. S. (2015). Importância das Disfunções Miccionais na Urologia. Visão Universitária. (3) 109-28. http://www.visaouniversitaria. com.br/ojs/index.php/home/article/viewFile/59/51

Arruda R. M. et al (2007). Hiperatividade do detrusor: comparação entre oxibutinina, eletroestimulação funcional do assoalho pélvico e exercícios perineais. Estudo randomizado. Revista Brasileira de Ginecologia e Obstetrícia, 29(9), 452-458.

Beuttenmüller, L. et al. (2011). Contração muscular do assoalho pélvico de mulheres com incontinência urinária de esforço submetidas a exercícios e eletroterapia: um estudo randomizado. Fisioterapia e Pesquisa, 18(3).

Benvenutti C. (1987). Reductive treatment of female genuine stress incontinence. Jounal of medical.

Burgio K. L. et al (1991). Prevalence, incidence and correlates of urinary incontinence in healthy, middle-aged women. O Journal of Urology, 146(5), 12551259 .

Brasil D. M. et al. (2018). Incontinência urinária e função sexual feminina: revisão integrativa de questionários validados. Acta Paulista Enfermagem, 31(5):558-63.

Caetano, A. S. \& Tavares, M. C. G. C. F. (2004). Proposta de atividades físicas para mulheres com incontinência urinária de esforço. Revista Digital. Buenos Aires, 10(76), 1.

Carvalho, M. Â. C. R \& Freitas, M. M. S. (2011). Aspectos conceituais da incontinência urinária de esforço, incontinência urinária de urgência e incontinência urinária mista. In: Ferreira, C. H. J. (Org.) Fisioterapia na Saúde da Mulher: teoria e prática. Editora Guanabara Koogan, 9(1), 60-63.

Castro A. P. et al (2010). Eficácia do biofeedback para o tratamento da incontinência urinária de esforço: uma revisão sistemática. Science Translational Medicine., 20(3), 257-263.

Castro, R. de A. et al. (2008). Fisioterapia e incontinência urinária de esforço: revisão e análise crítica. Revista Femina, 36(12), 737-742.

Delarmelindo R. C. A. et al (2013). Estratégias de enfrentamento da incontinência urinária por mulheres. Revista da escola de Enfermagem da USP, 47(2), 296-303.

Felicíssimo M.F. et al (2007). Fatores limitadores à reabilitação da musculatura do assoalho pélvico em pacientes com incontinência urinária de esforço. Acta Fisiátrica, 14(4), 233-236. 
Filho et al. (2013). Análise dos recursos para reabilitação da musculatura do assoalho pélvico em mulheres com prolapso e incontinência urinária. Fisioterapia Pesquisa, 20(1), 90-96.

Fitz F. et al (2012). Efeito da adição do biofeedback ao treinamento dos músculos do assoalho pélvico para tratamento da incontinência urinária de esforço. Revista Brasileira de Ginecologia e Obstetrícia, 34(11),505-10.

Fitz et al. (2012). Impacto do treinamento dos músculos do assoalho pélvico na qualidade de vida em mulheres com incontinência urinária. Revista de Associação Medica Brasileira ,58(2), 155-159.

Franco et al. (2011). Avaliação da qualidade de vida e da perda urinária de mulheres com bexiga hiperativa tratadas com eletroestimulação transvaginal ou do nervo tibial. Fisioterapia e Pesquisa, 18(2), 145-50.

Galhardo, C. \& Katayama, M. (2007). Anatomia e fisiologia do trato urinário inferior feminino. In: Chiarapa, T. R.; Cacho, D. P.; Alves, A. F. D. (Org.) Incontinência Urinária Feminina: assistência fisioterapêutica e multidisciplinar. Livraria Médica Paulista, 1(1), 8-25.

Gomes, P. A. G. P. N. (2010) Incontinência Urinária. Revista da Associação Médica Brasileira, 56(6).

Impey, L. (2007). Patologias do trato urinário: Obstetrícia e Ginecologia.: Editora Tecmedd.

Lopes M. H. B. M. et al (2017). Pelvic floor rehabilitation program: report of 10 years ofexperience. Revista Brasileira de Enfermagem, 70(1), 219-23.

Marques, A. de A. \& Ferreira, N. de O. (2011). Fisiologia da micção e fisiopatologia da incontinência urinária. In: Marques, A. de A.; Silva, M. P. e; Amaral, T. P. do. (Org.) Tratado de Fisioterapia em Saúde da Mulher. Editora Roca, 25(1), 269-270.

Matheus, L. M. et al. (2006). Influência dos exercícios perineais e dos cones vaginais, associados à correção postural, no tratamento da incontinência urinária feminina. Revista Brasileira de Fisioterapia, 10(4), 387-392.

Menezes G. M. D. et al. (2011) Queixa de perda urinária: um problema silente pelas mulheres. Revista Gaúcha de Enfermagem., Porto Alegre (RS);33(1):1008 .

Monteiro, M. V. de C. \& Silva Filho, A. L. (2007). Incontinência urinária. In: Baracho, E. (Org.) Fisioterapia Aplicada à Obstetrícia, Uroginecologia e Aspectos de Mastologia. Editora Guanabara Koogan, 4(26), 288.

Moreno, A. L. (2004). cinesioterapia do assoalho pélvico. Fisioterapia em Uroginecologia. Editora Manole.

Moreno, A. L. (2009). cinesioterapia funcional do assoalho pélvico. Fisioterapia em Uroginecologia. Editora Manole.

Nunes, P. L. \& Resplande, J. (2009). Fisiopatologia da incontinência urinária feminina. In: Palma, P. C. R. (Org.) Urofisioterapia: aplicações clínicas das técnicas fisioterapêuticas nas disfunções miccionais e do assoalho pélvico. Personal Link Comunicações, 1(4), 65.

Oliveira J.R \& Garcia R.R. (2011). Cinesioterapia no tratamento da incontinência urinária em mulheres idosas. Revista Brasileira de Geriatria e Gerontologia., 14(2), 343-351.

Peattie, A.B et al. (1988) Vaginal cones: a conservative method of treating genuine stress incontinence. Brit. Revista de obstetrícia e Ginecologia, 95,1049.

Pinheiro B.F et al. (2012). Fisioterapia para consciência perineal: uma comparação entre as cinesioterapias com toque digital e com auxílio do biofeedback. Fisioterapia do. Movimento, 25(3), 639-648.

Polden M. \& Mantle J. (2002). Fisioterapia em Ginecologia e Obstetrícia. Editora Santos.

Portugal, H. S. P. \& Palma, P. C. R. (2009). Anatomia do assoalho pélvico. In: Palma, P. C. R. (Org.) Urofisioterapia: aplicações clínicas das técnicas fisioterapêuticas nas disfunções miccionais e do assoalho pélvico. Personal Link Comunicações, 1(1), 27.

Ramos, L. (2014). RPA: reabilitação perineal ativa. Editora Scribd, 1, 1-87.

Rizzi, S. (2012). Incontinência Urinária de Urgência.

Rother, E. T. (2017). Revisão sistemática X revisão narrativa. Acta Paulista de Enfermagem, 20(2), 5-6.

Rubinstein, I. et al. (2000). Urologia Feminina. Editora BYK.

Smeltzer, S. C. \& Bare, B. G. (2005). Histórico das funções renal e urinária. Brunner \& Suddarth, Tratado de Enfermagem Medico-Cirúrgica. 2 vols. Editora Guanabara Koogan.

Spence, a. P. (1991). Sistema Urinário. Anatomia Humana Básica. Editora Manole. 